\title{
Advancements of the Lightweight Integrated Solar Array and Transceiver (LISA-T) Small Spacecraft System
}

\author{
Tiffany Russell ${ }^{1}$, Armando Martinez ${ }^{1}$, Darren Boyd ${ }^{1}$, Michael SanSoucie ${ }^{1}$, Brandon Farmer ${ }^{2}$, Todd \\ Schneider ${ }^{1}$, Leo Fabisinski ${ }^{1}$, Les Johnson ${ }^{1}$, and John A. Carr ${ }^{1}$ \\ ${ }^{1}$ National Aeronautics and Space Administration (NASA), Marshall Space Flight Center (MSFC), \\ Huntsville, Al, 35812, U.S.A \\ ${ }^{2}$ ManTech NeXolve Corporation, Huntsville, Al, 35806, U.S.A
}

\begin{abstract}
This paper describes recent advancements of the Lightweight Integrated Solar Array and Transceiver (LISA-T) currently being developed at NASA's Marshall Space Flight Center. The LISA-T array comprises a launch stowed, orbit deployed structure on which thin-film photovoltaic (PV) and antenna devices are embedded. The system provides significant electrical power generation at low weights, high stowage efficiency, and without the need for solar tracking. Leveraging high-volume terrestrial-market PVs also gives the potential for lower array costs. LISA-T is addressing the power starvation epidemic currently seen by many small-scale satellites while also enabling the application of deployable antenna arrays. Herein, an overview of the system and its applications are presented alongside sub-system development progress and environmental testing plans / initial results.
\end{abstract}

\section{INTRODUCTION}

The trend of satellite miniaturization continues to enable lower-cost alternatives to the traditional large-scale spacecraft; opening a door to space for many research and commercial payloads. These small-scale satellites, however, are inherently plagued by limited surface area, volume, and mass allocation; limiting solar array real-estate and electrical power generation. Solar array deployment options exist, but are still limited in size and some levy the requirement of solar tracking. Thus, though a lower-cost vehicle to space exists, capabilities remain choked by the small power systems.

The use of thin-film based solar arrays for spacecraft applications has long been recognized as an advantageous power generation option. [1] Thinner materials yield a mass savings, equating to lighter launch loads or more payload allocation. Furthermore, their mechanical flexibility lends itself well to stowage and deployment schemes. Both make thin-film arrays an exciting prospect for small-scale satellites. However, a gap in thin-film array development exists, leaving very few choices for available array structures. [1] The Lightweight Integrated Solar Array and Transceiver (LISA-T) seeks to address this, enabling higher power generation in small-scale satellites at low weights, high stowage efficiency, and without the need for solar tracking.
LISA-T is a scalable, launch stowed, orbit deployed array on which thin-film solar power and communication devices are be embedded (Fig. 1). Initial estimates indicate upwards of $200 \mathrm{~W}$ of power generation can be packed/deployed from $1 \mathrm{U}$ and nearly $500 \mathrm{~W}$ from $2 \mathrm{U}$. Furthermore, the system can also leverage high-volume terrestrial market photovoltaics (PVs), lowering module costs $(\$ / W)$. The integration of thin-film antennas allows communication arrays to be simultaneously deployed. An option for '3-dimensional deployment' eliminates the need for solar tracking and allows for stronger omnidirectional communications.

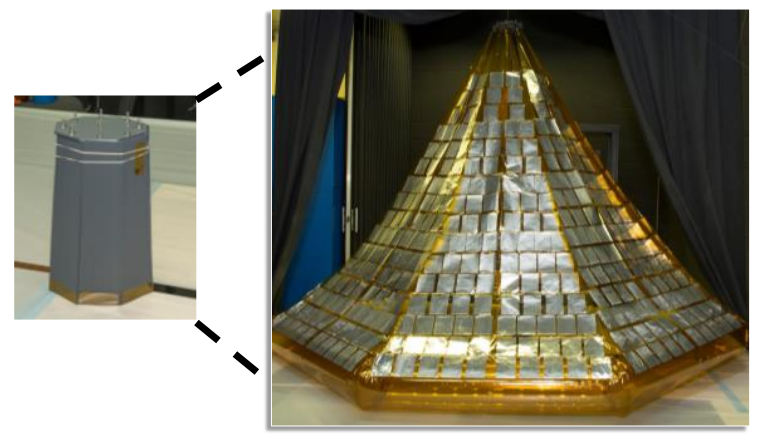

Fig. 1. Internal MSFC Technology Investment Program (TIP) TRL4 demonstration of a conceptual LISA-T design in 2012.

LISA-T builds upon previously published concepts, such as the PowerSphere [2], utilizing new engineering concepts and the latest advancements in thin-film materials, devices, and deployment options. The project leverages several existing and on-going efforts at Marshall Space Flight Center (MSFC) for design, development, fabrication, and test. The most current LISA-T activity will advance the system technology readiness level (TRL) from 4 through 6+. The project is supported through a NASA space technology development activity called the Early Career Initiative (ECI) sponsored by the Space Technology Mission Directorate (STMD) at NASA Headquarters. The project is being completed in partnership with ManTech $\backslash$ NeXolve (Huntsville, Al, U.S.A) and is being managed under the Small Spacecraft Technology (SST) 
TABLE I

SUMMARY OF GEOMETRY SIZING TRADE: TOTAL DEPLOYED ARRAY TO GENERATE 200W POWER FROM 25\% PV.

\begin{tabular}{|c|c|c|c|c|}
\hline Shape & $\begin{array}{c}\text { Packing } \\
\text { density }(\%)\end{array}$ & $\begin{array}{c}\text { Area } \\
\left(\mathbf{m}^{\mathbf{2}}\right)\end{array}$ & $\begin{array}{c}\text { Configuration } \\
\text { Factor }\end{array}$ & $\begin{array}{c}\text { Total Area } \\
\text { Required }\left(\mathbf{m}^{\mathbf{2}}\right)\end{array}$ \\
\hline Cube & 90 & 0.65 & 5.00 & 3.25 \\
\hline Pyramid & 80 & 0.79 & 3.00 & 2.36 \\
\hline Cylinder & 80 & 0.92 & 3.14 & 3.61 \\
\hline Sphere & 80 & 0.92 & 4.00 & 3.66 \\
\hline $3 / 4$ Torus & 80 & 0.92 & 2.00 & 1.83 \\
\hline
\end{tabular}

*DOES NOT INCLUDE CIRCULAR TOP

program. Herein, the most recent concept(s) and design progress of the LISA-T platform will be discussed.

\section{GEOMETRY AND DEPLOYMENT}

A step being taken in the development of LISA-T is an option to maintain a constant power generation independent of the incident angle - a '3-dimensional' deployment to simplify Guidance, Navigation, and Control by eliminating the need for array or spacecraft solar pointing. A trade study has commenced to investigate various deployment geometries, which include a cube, cylinder, sphere, triangular pyramid, and torus. Each shape is effected differently by potential packing densities, the configuration knockdown factor (related to projected area and number of deployed sides), as well as the Kelly Cosine Law (Table I). For example, a square side of a cube resembles a more traditional solar panel and cells can be packed to cover more of the surface area $(90 \%+)$. With an average power generation from a $25 \%$ efficient cell of $\sim 307$ $\mathrm{W} / \mathrm{m}^{2}$ at the module level, $\sim 0.65 \mathrm{~m}^{2}$ must be illuminated at any given time to produce $200 \mathrm{~W}$ of continuous power. To configure this into a cube 5 or 6 sides must be deployed simultaneously to eliminate the need for pointing and, hence, at least $3.25 \mathrm{~m}^{2}$ of array must be stowed/deployed. Worst case Kelly Cosine losses are when illumination is normal to one side, as angled incidence will illuminate multiple sides generating more power. Similar arguments for other geometries can be made and will be presented.

To further down select the geometries, device (antenna and PV) integration along with the ease of fabrication, stowage, deployment, and the like must be considered. Results of this trade study will also be presented.

Various types of deployment methods will also be reviewed. Mechanical 'boom-type', inflatable, and hybrid options are currently being explored. Some key factors for selection are stiffness, mass, TRL, reliability, stowed volume and scalability. The current LISA-T development effort will produce a geometric and mechanical design by the date of the conference. The intention will be to share the trade study results and design implications.

\section{PHOtOVOLTAIC AND MATERIALS}

Of critical importance to the system are the thin-film PV cells. The field of thin-film photovoltaics is somewhat broad and encompasses technologies from the more traditionals such amorphous silicon (a:Si) or copper indium gallium (di)selenide (CIGS) to the emerging such as perovskites. Several technologies can be utilized by LISA-T each with their own distinct advantages. A metric based matrix is currently being built to offer building blocks for different types of missions. For example, a high performance/reliability mission (superior $\mathrm{W} / \mathrm{m}^{2}, \mathrm{~W} / \mathrm{m}^{3}, \mathrm{~W} / \mathrm{kg}$, and lifetime) with a higher budget ( $\$ / \mathrm{W}$ allocation) can be envisioned. Spacequalified $>30 \%$ inverted metamorphic (IMMs) may be best suited for this application. In contrast, a lower budget mission with less emphasis on performance metrics might also be envisioned. Adapted terrestrial market, roll-to-roll CIGS may be best suited. Of course, each option will still enable higher total power generation than current state of the art. Partnerships with various PV manufactures are currently being built; specific technologies, configurations, and test data will be reported.

Deployment materials are also being heavily considered. Most viable is colorless polyimide 1 (CP1) produced by ManTechlNexolve. This material opens the door for parameter tune-ability (e.g. emissivity / alpha) and the ability to embedded bus bars and cells to cell interconnections. Cell cover protectant materials also remain an open option. Several are being considered, but initial trades indicate short term missions may be better off flying bare. Results of the materials trade study will be presented.

\section{TRANSCEIVER}

Solar cells and antenna structures often compete for valuable surface area, volume and mass allocation - especially in small-scale space systems. The objective of the LISA-T transceiver subsystem is to integrate solar cell arrays and antenna elements onto one three dimensional structure. This will save resources by sharing deployment hardware, eliminate mechanical pointing, and create more antenna real-estate. Incorporating antenna elements into multiple positions on a three dimensional deployable structure will allow for both omnidirectional communication and possible phase array steering.

Spacecraft systems vary across a wide range of frequencies and bands. Various antenna types and radio frequency (RF) bands are being investigated and evaluated for optimal efficiency in both communication and integration. These bands include but are not limited to UHF/VHF, S-band, and X-band. Pole, patch, and slot antennas are being 
evaluated. Stand alone and phase array implementations are also being explored. The antenna structures may be integrated into the deployment support structures or interspersed among the solar cells. Initial designs and trades will be reported.

\section{TESTING}

The LISA-T project will involve a significant amount of testing. A life-cycle test bed is being built and a considerable amount of space environmental effects testing will be performed at MSFC.

The life-cycle test bed will support the testing of custom, small-satellite power systems. It allows integrated power systems to be tested as per expected orbit, in a space environment with real-time simulated zero atmosphere (AM0) solar illumination, power load profiles, and electrical characterization. Using liquid nitrogen cooled cold plates, thermal cycling will also be possible. All of this makes the test bed valuable for development and testing of advanced power system research (e.g. LISA-T).

A test plan for environmental effects testing is also being developed. Testing such as particulate radiation, UV radiation, angle of incidence, solar environment, and atomic oxygen is being developed by tailoring portions current solar cell and panel standards. Details and initial test data will be presented.

\section{CONCLUSION}

The LISA-T project is an innovative approach to integrating a small satellite's solar array and transceiver elements. The design will be scalable and adaptable to mission requirements. The technology will enable small satellites to perform more in-depth science with the availability of greater electrical power and communication arrays. Extensive work is currently underway designing the geometric shape, the deployment mechanisms, stowage, testing the state of the art thin film PVs and transceiver elements, and substrate development. The LISA-T will receive a battery of space environments tests on the component level as well as at the integrated system level. At the end of the current two year effort a fully tested, TRL 6 qualified engineering unit will be developed. Advancements, design configurations, and initial test data of this effort will be presented.

\section{REFERENCES}

[1] S. Bailey et al, "The Future of Space Photovoltaics," in Photovoltaic Specialists Conference (PVSC), 2009, p. 1909.

[2] E. Simburger et al, "Thin Film Technology Development for the PowerSphere," Materials Science and Engineering: B, 2005, Vol. 116, Iss. 3, p. 265-272. 